\title{
Die SGPH: Expertennetzwerk im Einsatz gegen pulmonale Hypertonie
}

\section{Frédéric Lador ${ }^{a}$, Andrea Azzolab ${ }^{b}$ Silvia Ulrich ${ }^{c}$}

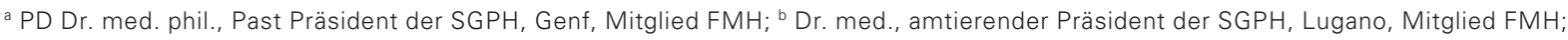

${ }^{c}$ Prof. Dr. med., Lead Scientific Committee der SGPH, Zürich, Mitglied FMH

\author{
Die Schweizerische Gesellschaft für Pulmonale Hypertonie (SGPH) feiert ihr zwan- \\ zigjähriges Bestehen. Dieses freudige Ereignis möchten wir zum Anlass nehmen \\ für eine Reflexion über ihre Geschichte und Entwicklung, aktuelle Projekte und die \\ Krankheit, der sie sich seit zwei Jahrzehnten ausschliesslich widmet.
}

\section{Mit Interdisziplinarität zum Erfolg}

Die pulmonale Hypertonie ist eine Krankheit, die sich durch einen erhöhten Blutdruck in den Lungenarterien präsentiert. Um den fatalen Folgen der pulmonalen Hypertonie in der Schweiz möglichst entgegenzuwirken und die Behandlungsqualität für die Patientinnen und Patienten mit PH zu steigern, wurde 1998 die Schweizerische Gesellschaft für Pulmonale Hypertonie (SGPH) gegründet. Pneumologen, Intensivmediziner, Angiologen, Kardiologen, Pädiater und Internisten gehörten zu den Initianten der neuen Gesellschaft - diese interdisziplinäre Prägung der SGPH ist ihr bis heute als wichtiges Merkmal erhalten geblieben: Unsere Gesellschaft besteht heute aus knapp 90 Mitgliedern aus den unterschiedlichsten Disziplinen, die sich in der SGPH nach wie vor alleine diesen teilweise seltenen Erkrankungen widmen, welche eine pulmonale Hypertonie auslösen.

\section{Austausch und Förderung}

Die Ziele der SGPH haben sich seit Beginn nicht geändert. Wir sehen es noch immer als unsere grösste und wichtigste Aufgabe, die Behandlungsqualität von Patientinnen und Patienten mit unterschiedlichen Arten der pulmonalen Hypertonie in der Schweiz zu gewährleisten, aber auch zu verbessern, unter anderem, indem wir die Ärzteschaft in unserem Land, aber auch die Öffentlichkeit auf diese Erkrankung aufmerksam machen. Zu diesem Zweck erhalten mehrere Tausend Ärztinnen und Ärzte unterschiedlichster Disziplinen zweimal jährlich einen gedruckten Newsletter, der seit vielen Jahren auch in digitaler Form erscheint - die Lektüre lohnt sich für Ärzte und Ärztinnen der unter- schiedlichsten Fachgebiete. Diese Publikation widmet sich seit 15 Jahren auf vier oder gar acht Seiten einem oder mehreren Schwerpunktthemen zur pulmonalen Hypertonie. Nicht selten gelingt es uns, darin auch internationale Expertinnen und Experten zu Wort kommen zu lassen. Die Inhalte sind vielfältig, jeder Newsletter widmet sich vertieft einem Thema im Bereich der pulmonalen Hypertonie mit Informationen zum neuesten Stand der Forschung und Therapie. Aus Anlass des 20-jährigen Bestehens haben wir unseren SGPH-Newsletter in diesem Jahr äusserlich modernisiert.

Der wissenschaftliche Austausch zwischen einheimischen und internationalen Expertinnen und Experten wird an den Workshops und Kongressen unserer Gesellschaft gefördert. Am 8. und 9. November dieses Jahres trafen sich im Palais de l'Athénée in Genf Expertinnen und Experten auf dem Gebiet der pulmonalen Hypertonie zum siebten internationalen Kongress der SGPH.

Die Publikation im SGPH-Newsletter oder die Präsentation eines aktuellen Projekts an einer unserer Veranstaltungen sind für unsere Gesellschaft aber nicht die einzigen Wege, wertvolle Forschungstätigkeiten im Bereich der pulmonalen Hypertonie zu würdigen. Seit einiger Zeit vergibt die SGPH Forschungspreise und -stipendien an Wissenschaftlerinnen und Wissenschaftler jeden Alters, die sich besonders intensiv und erfolgreich mit der pulmonalen Hypertonie beschäftigen.

\section{Swiss PH Registry}

Die SGPH trägt als Gesellschaft weiter zur Forschung auf dem Gebiet der pulmonalen Hypertonie bei, indem 
sie präzise epidemiologische Daten sammelt, um auf diese Weise ein aktuelles Register für unterschiedliche Formen und Schweregrade der pulmonalen Hypertonie in der Schweiz aufzubauen, das dazu dient, mehr über die Krankheit zu erfahren. Im Jahr 1998 wurde das «Swiss PH Registry» in Betrieb genommen und wird seither ständig erneuert. Eine grosse Revision fand im Jahre 2014 statt.

\section{Swiss CTEPH Board}

Seit Januar 2018 ist über die SGPH eine neue Plattform des Austausches zwischen Ärztinnen und Ärzten für die Patientinnen und Patienten installiert: das Schweizerische Board für Chronisch-Thromboembolische Pulmonale Hypertonie (CTEPH). Dieses wird von interdisziplinären Ärzteteams der Universitätsspitäler Zürich (USZ) und Genf (HUG) geleitet, die sich seit Jahren mit dieser bestimmten Form der pulmonalen Hypertonie beschäftigen. Kollegen aus anderen Universitätsspitälern, Kantonsspitälern und Praxen können sich zuschalten und nach Anmeldung per E-Mail (cteph[at] sgph.ch) ihre Patientenfälle vorstellen. Die Einzelheiten des Patientendossiers werden per Videokonferenz geteilt, wodurch Spezialisten der Pneumologie, Kardiologie, Radiologie, Thoraxchirurgie und weiterer Fächer ermöglicht wird, die Problematik der Patientinnen und Patienten interdisziplinär zu besprechen und eine gemeinsame Empfehlung zur weiteren Behandlung abzugeben.

Korrespondenz:

Schweizerische Gesellschaft für Pulmonale Hypertonie SGPH

c/o IMK Institut für Medizin und Kommunikation AG

Münsterberg 1

CH-4001 Basel

ssph[at]imk.ch

\section{Empfehlungen und Beratungen}

Es ist uns ausserdem ein Anliegen, wissenschaftlich abgestützte Diagnose- und Behandlungsrichtlinien zu erarbeiten und diese regelmässig zu aktualisieren. In Zusammenarbeit mit einer Arbeitsgruppe der Schweizerischen Gesellschaft für Kardiologie (SGK) haben wir erstmals Empfehlungen publiziert, die unseren ärztlichen Kolleginnen und Kollegen dabei behilflich sein sollen, eine transthorakale Echokardiographie durchzuführen, um so bei Patientinnen und Patienten eine mögliche pulmonale Hypertonie festzustellen und zu bewerten. Natürlich stehen die Expertinnen und Experten der SGPH auch in einem Konsilium bei der Behandlung von Patientinnen und Patienten mit pulmonaler Hypertonie gerne zur Verfügung. Diese Beraterfunktion übt die SGPH auch bei Patientenorganisationen für pulmonale Hypertonie regelmässig aus.

Die Hauptaufgaben der SGPH werden auch in Zukunft sein, die Bewusstseinsbildung für ein potentiell fatales Krankheitsbild weiter zu steigern und die Behandlungsqualität für Patientinnen und Patienten mit pulmonaler Hypertonie in der Schweiz auf höchstem Niveau zu halten. Dazu gehören regelmässige Kontrollen durch die betreuenden Ärzte, Spezialisten und Zentren für pulmonale Hypertonie sowie die Überprüfung der erreichten Therapieziele und gegebenenfalls eine Therapieanpassung. Um den Standard für die Behandlung einer seltenen Lungenerkrankung hochzuhalten, ist eine gute interdisziplinäre Zusammenarbeit in wenigen Expertenzentren erforderlich. Die SGPH, mit ihren Mitgliedern aus verschiedenen Fachgebieten, wird sich im Interesse der Patientinnen und Patienten auch weiterhin für die Kooperation mit anderen Fachgesellschaften engagieren.

Weitere Informationen zur pulmonalen Hypertonie, zur SGPH, zu aktuellen Projekten und Veranstaltungen finden Sie auf www. sgph.ch. 